\title{
Classical $A b$ Initio Molecular Dynamics Run at an Elevated Temperature is Not a Good Model for the Nuclear Quantum Effects in Water at Ambient Temperature
}

Chenghan Li, ${ }^{1}$ Francesco Paesani, ${ }^{2,}{ }^{*}$ and Gregory A. Voth ${ }^{1, *}$

${ }^{1}$ Department of Chemistry, Chicago Center for Theoretical Chemistry, James Franck Institute, and Institute for Biophysical Dynamics, The University of Chicago, Chicago, IL, 60637

${ }^{2}$ Department of Chemistry and Biochemistry, Materials Science and Engineering, and San Diego Supercomputer Center, University of California San Diego, La Jolla, California 92093,

*Corresponding Author: fpaesani@ucsd.edu, gavoth@uchicago.edu

\section{ABSTRACT}

It is a common practice in ab initio molecular dynamics (AIMD) simulations of water to use an elevated temperature to overcome the over-structuring and slow diffusion predicted by most current density functional theory (DFT) models. The simulation results obtained in this distinct thermodynamic ensemble are then compared with experimental data at ambient temperature based on the rationale that a higher temperature effectively recovers nuclear quantum effects (NQEs) that are missing in the classical AIMD simulations. In this work, we systematically examine the foundation of this assumption for several DFT models as well as for the many-body MB-pol model. We find for the cases studied that a higher temperature does not correctly mimic NQEs at room temperature, which is especially manifest in significantly different three-body correlations as well as dynamics. In many of these cases, the effects of NQEs are exactly the opposite of the effects of carrying out the simulations at an elevated temperature.

\section{INTRODUCTION}

Due to the light mass of hydrogen nuclei, nuclear quantum effects (NQEs) are sometimes assumed to be important for a quantitative modeling of the structural, thermodynamic, and dynamical properties of liquid water. ${ }^{1}$ Though NQEs appear to be minimal for the calculated intermolecular properties in some water simulations, ${ }^{2,3}$ they were found to be non-negligible in other cases ${ }^{4,5}$ as demonstrated by the differences in thermodynamic properties between light and heavy water. ${ }^{1}$ 
In principle, Feynman's imaginary-time path-integral formalism ${ }^{6}$ enables the modeling of NQEs in liquid water to numerical accuracy, but the associated high computational cost has hindered widespread application of path-integral molecular dynamics (PIMD) simulations until recent developments of more efficient approximations, ${ }^{7}$ such as the ring-polymer contraction (RPC), ${ }^{8}$ the ring-polymer interpolation, ${ }^{9}$ and the combined path-integral and generalized Langevin equation (PI+GLE) approach. ${ }^{10}$ The computational cost of a PIMD simulation of liquid water significantly increases when the underlying Born-Oppenheimer potential energy surface is calculated "on the fly" as in ab initio molecular dynamics (AIMD) simulations ${ }^{11}$ where Kohn-Sham density functional theory ${ }^{12}$ (KS-DFT) is generally used to solve the (electronic) Schrödinger equation at each step of the dynamical trajectory. As a consequence, most of the AIMD simulations reported in the literature have been conducted ignoring NQEs and treating the nuclei as classical particles.

Among existing exchange-correlation functionals, generalized-gradient approximation (GGA) functionals have been extensively used in AIMD simulations of liquid water due to their relatively lower computational cost. GGA functionals typically overestimate the strength of the hydrogen bonds in water. This results in over-structuring of the liquid phase which is accompanied by slow molecular diffusion and, in some cases, glassy-like behavior. ${ }^{13}$ The inclusion of dispersion corrections was found to partially alleviate these problems. ${ }^{13}$ An alternative and ad hoc approach adopted in the literature to overcome over-structuring and slow diffusion in AIMD simulations of liquid water consists of performing the simulations at a higher temperature. While simulations at a higher temperature sample a different thermodynamic ensemble, it is sometimes implicitly assumed that the extra thermal energy effectively mimics NQEs that are missing from the classical AIMD simulations at room temperature. Within this assumption, the results obtained from classical AIMD simulations carried out at higher temperature are thus sometimes considered to be equivalent to the actual results at room temperature with NQEs included.

Although there has been discussion on the temperature dependence of NQEs for empirical and data-driven water models, ${ }^{14-17}$ a rigorous justification for the use of elevated-temperature AIMD simulations to recover NQEs has not been systematically established or examined. Herein, we therefore employ the RPC method to explicitly model NQEs at room temperature and systematically benchmark NQEs on structural correlations as well as dynamical properties of liquid water at room temperature against classical molecular dynamics (MD) simulations carried 
out at higher temperature. Our analysis includes three exchange-correlation functionals: (1) the strongly constrained and appropriately normed (SCAN) functional, a meta-GGA functional, ${ }^{18}$ (2) the Becke-Lee-Yang-Parr functional ${ }^{19,}{ }^{20}$ with D3 dispersion correction ${ }^{21}$ (BLYP-D3), one of the most common GGA functionals, and (3) the BLYP-D3 with the experimental directed simulation (EDS) correction. ${ }^{22}$ The EDS correction employs a minimal bias to improve the BLYP-D3 description of hydrogen bonding in liquid water, and has been shown to provide significantly more accurate water properties. ${ }^{22}$ Additionally, we perform the same analyses using MB-pol, ${ }^{23-25}$ arguably the most accurate water potential developed to date. MB-pol is a data-driven model rigorously derived from the many-body expansion of the interaction energies calculated at the coupled cluster level of theory - a model which has been shown to accurately predict the properties of water, from small gas-phase clusters to liquid water and ice. ${ }^{26,27}$ We note that the goal of this present study is not to determine which water model and simulation protocol best reproduces the experimental data, but to assess the validity of using an elevated temperature in classical MD simulations of liquid water to effectively model NQEs at ambient temperature.

\section{METHODS}

The BLYP-D3 and EDS-BLYP-D3 simulations were performed with 128 water molecules in a cubic simulation box of side $L=15.64 \AA$. The Goedecker-Teter-Hutter (GTH) pseudopotentials ${ }^{28}$ were used to model the core electrons, while a TZV2P basis set was used to expand the KohnSham orbitals and a plane wave basis set with a cutoff of 400 Ry was used to expand the electron density. The orbital transformation (OT) method $^{29}$ was used to optimize the wave function at each step, using a self-consistent field (SCF) convergence criterion of $1 \times 10^{-7}$ a.u. The SCAN simulations were performed with 64 water molecules in a cubic box of side $\mathrm{L}=12.66 \AA$ to be consistent with the setup in Ref. ${ }^{30}$. Similar to the BLYP-D3 simulations, the TZV2P basis set was used but a larger plane wave basis set with a cutoff of 600 Ry was used for better SCF convergence. The GTH pseudopotentials optimized for SCAN were used to model the core electrons (https://github.com/juerghutter/GTH). The OT method was used to optimize the wave function and the SCF convergence criterion was the same as BLYP-D3.

For the classical AIMD simulations with BLYP-D3 and EDS-BLYP-D3, the system was equilibrated in the canonical (constant NVT) ensemble for 90 ps at $298 \mathrm{~K}$ and for 85 ps at $328 \mathrm{~K}$. 
In the case of SCAN, the NVT equilibration was carried out for 70 ps at $298 \mathrm{~K}$ and for 72.5 ps at 328 K. In all simulations, a Nosé-Hoover chain thermostat with a characteristic frequency of 3000 $\mathrm{cm}^{-1}$ was coupled to all degrees of freedom, and a timestep of $0.5 \mathrm{fs}$ was used to integrate Newton's equations of motion. All simulations were carried out with the $\mathrm{CP} 2 \mathrm{~K}$ software package. ${ }^{31,32}$ The EDS correction was added via a modified version of PLUMED2 ${ }^{33}$ coupled to CP2K, with the EDS parameters taken from Ref. ${ }^{22}$, while the simulations with the SCAN exchange-correlation functional were carried out by linking the Libxc library ${ }^{34}$ to CP2K.

The dynamical properties were calculated by performing MD simulations in the microcanonical (constant NVE) ensemble which were started from configurations previously equilibrated in the canonical ensemble. At each temperature, five independent replicas were launched from the NVT trajectories at intervals of $10 \mathrm{ps}$, and were carried out for 30-40 ps. The multi-time stepping (MTS) scheme was used to accelerate the simulations, with an inner timestep of $0.25 \mathrm{fs}$ and an outer timestep of 2 fs. For the reference potential used in the MTS scheme, we used a deep learning potential (DP) trained on BLYP-D3, EDS-BLYP-D3, and SCAN data. The training set for BLYPD3 contained 2500 configurations sampled at $298 \mathrm{~K}$ and 2500 configurations sampled at $328 \mathrm{~K}$. The training set for EDS-BLYP-D3 contained 132000 configurations sampled at $298 \mathrm{~K}$. The training set for SCAN contained 10000 configurations sampled at $298 \mathrm{~K}$ and 10000 configurations sampled at $328 \mathrm{~K}$. Both energies and forces were used in the training. The training was conducted using the DeepMD-kit tool ${ }^{35}$ with the smooth edition of deep potential molecular dynamics $(\mathrm{DPMD})^{36}$. The BLYP-D3 energies and forces were calculated with CP2K, the forces for the DP potential were computed using LAMMPS $^{37}$ coupled to DeepMD-kit, the EDS correction was computed using PLUMED2, and the MD simulations were performed using the i-Pi force engine. ${ }^{38}$ The structural properties obtained from NVE simulations were found to be identical with those obtained in the NVT ensemble. An independent NVE simulation was carried out with a timestep of $0.5 \mathrm{fs}$, without applying the MTS approximation, to calculate the diffusion coefficient which was found to be identical to the value obtained from an analogous simulation carried out using the MTS scheme.

Approximate quantum dynamics simulations were carried out using thermostatted ring-polymer molecular dynamics (TRPMD). ${ }^{39}$ Following Ref. ${ }^{5}$, the RPC approach was employed with $P^{\prime}=1$, i.e., the centroid contraction. The path integral of each atom was discretized with $P=30$ beads, 
and all the analyses were performed on the centroid coordinates. The same reference potential used in MTS was used in RPC. Five independent TRPMD trajectories of 30-40 ps each were performed to calculate the dynamical properties. Classical and quantum MB-pol trajectories were taken from Refs. ${ }^{25,26,40 .}$
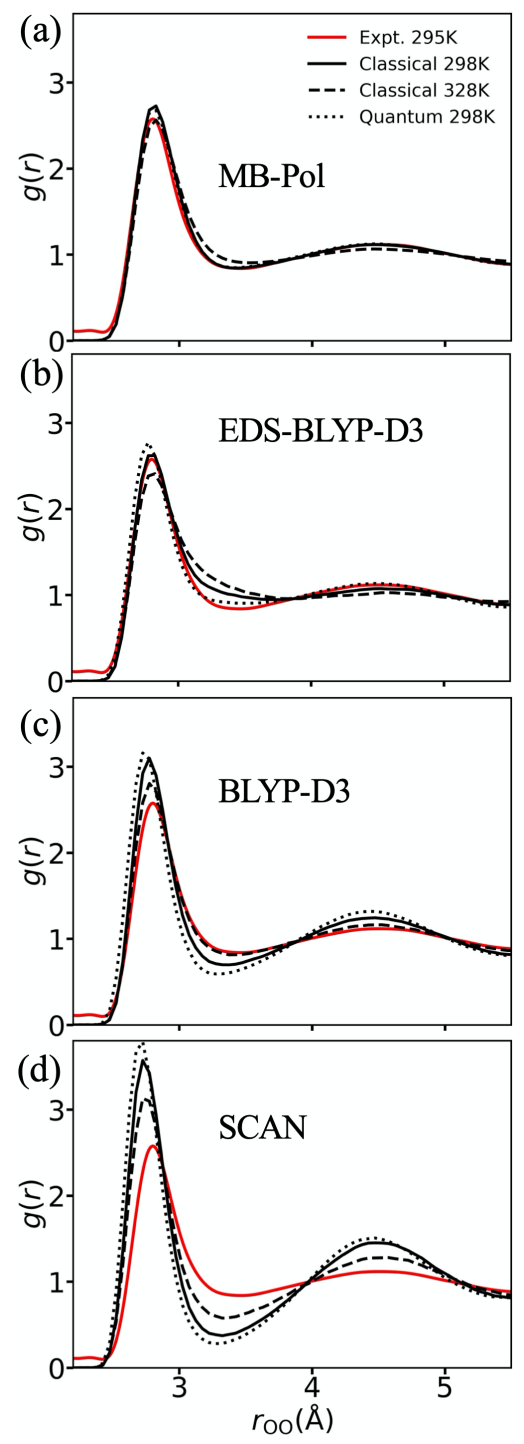

Figure 1. O-O RDFs of (a) MB-pol, (b) EDS-BLYP-D3, (c) BLYP-D3 and (d) SCAN water model at $298 \mathrm{~K}$ with classical nuclei (solid) and with quantized nuclei (dotted), and at $328 \mathrm{~K}$ (dashed). The experimental value ${ }^{41}$ at $295 \mathrm{~K}$ is plotted in red. 

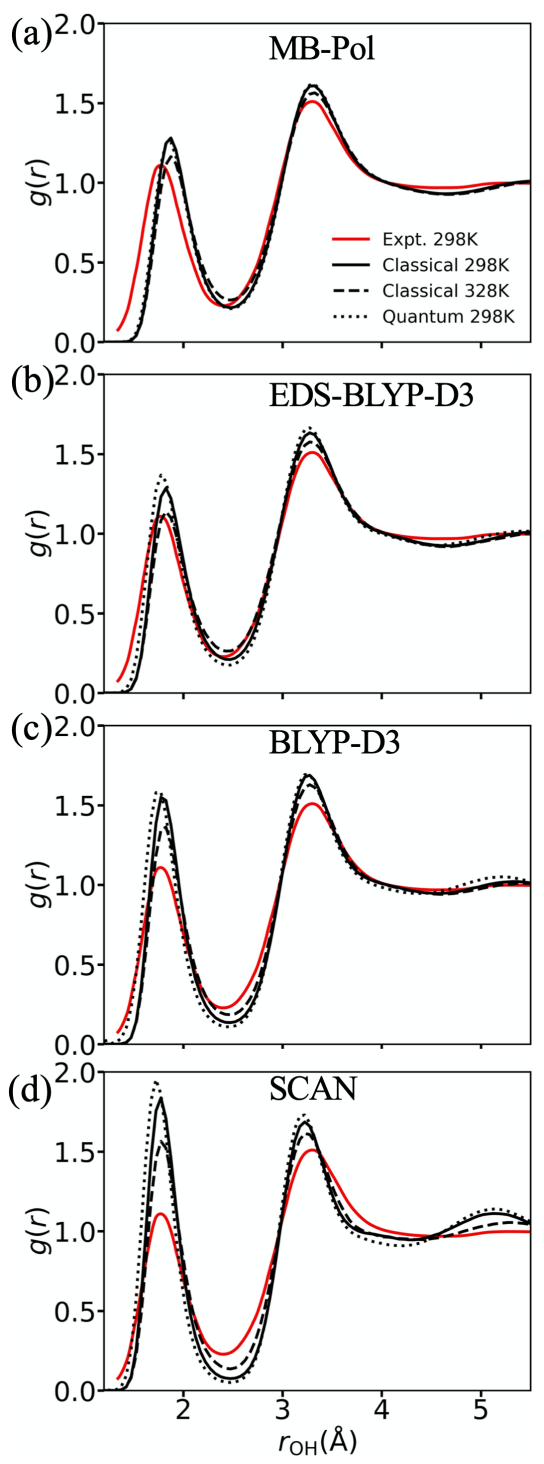

Figure 2. O-H RDFs of (a) MB-Pol, (b) EDS-BLYP-D3, (c) BLYP-D3 and (d) SCAN water model at $298 \mathrm{~K}$ with classical nuclei (solid) and with quantized nuclei (dotted), and at $328 \mathrm{~K}$ (dashed). The experimental value ${ }^{42}$ at $298 \mathrm{~K}$ is plotted in red. 

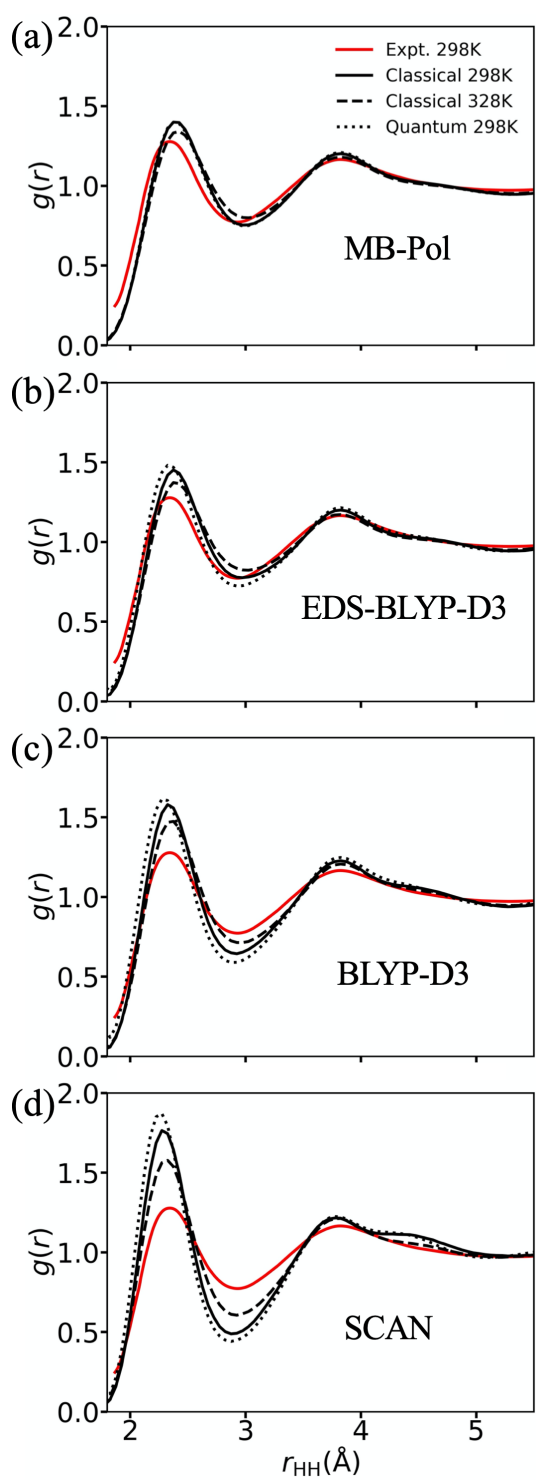

Figure 3. H-H RDFs of (a) MB-Pol, (b) EDS-BLYP-D3, (c) BLYP-D3 and (d) SCAN water model at $298 \mathrm{~K}$ with classical nuclei (solid) and with quantized nuclei (dotted), and at $328 \mathrm{~K}$ (dashed). The experimental value ${ }^{42}$ at $298 \mathrm{~K}$ is plotted in red.

\section{RESULTS AND DISCUSSION}

We analyzed simulations from the four water models (BLYP-D3, EDS-BLYP-D3, SCAN, and MB-pol) at ambient temperature $(298 \mathrm{~K})$ and at an elevated temperature $(328 \mathrm{~K})$ commonly used to effectively mimic NQEs. The simulations at $298 \mathrm{~K}$ were performed with both classical and quantized nuclei, while classical nuclei were used at $328 \mathrm{~K}$. 
Figure 1Figure 3 show the radial distribution functions of the four water models. As seen in Figure 1(a)-Figure 3(a), NQEs only have a minimal effect on these two-body correlations in MB-pol water, with the first peak in the O-O RDF being slightly less structured with quantized nuclei, which, in turn, slightly improves the agreement with the experimental data. NQEs show an opposite effect in the three DFT models where all the O-O, O-H, and $\mathrm{H}-\mathrm{H}$ RDFs become more structured with quantized nuclei. Notably, when NQEs are explicitly accounted for, the hydrogen bonds in DFT water shrink as shown by the first peak in the O-H RDFs (Figure 2(b)-(d)) moving towards shorter distances (dotted vs. solid curves). This agrees with the experimental observation of shorter hydrogen bond length in light water than heavy water. ${ }^{43}$ On the other hand, the effect of elevated temperature is consistent among the four water models - the extra thermal energy reduces the solvation structure in all the RDFs. It follows that performing classical MD simulations at an elevated temperature apparently mimics NQEs in simulations with MB-pol, while has opposite effects in simulations with the three DFT models. In fact, NQEs make the RDFs calculated with BLYP-D3 and SCAN even more structured, which is consistent with the more structured water seen in quantum AIMD simulations with a GGA functional reported in Ref. ${ }^{5}$. It should be noted that including NQEs improves the agreement between the EDS-BLYP-D3 and experimental RDFs.

We next examine three-body correlations in water by computing the tetrahedral order parameter $q$ defined as ${ }^{44}$

$$
q=1-\frac{3}{8} \sum_{i \neq j}\left(\cos \theta_{i j}+\frac{1}{3}\right)^{2},
$$

where $\theta_{i j}$ is the $\mathrm{O}_{i}-\mathrm{O}-\mathrm{O}_{j}$ angle centered on a given oxygen $\mathrm{O}$, and the sums are over the four closest oxygen atoms around 0 . The value of $q$ provides a measure of tetrahedral order, with a value of 1 corresponding to a perfect tetrahedral arrangement and a value of 0 representing the ideal gas limit. Figure 4 summarizes the distribution of $q$ for the four water models. NQEs still play a small role in determining the three-body correlations in the MB-pol simulations at ambient temperature. However, more pronounced differences are found in the simulations with the three DFT models, with the peak at $q \approx 0.85$ increasing and the peak at $q \approx 0.5$ decreasing, which is consistent with the more structured RDFs observed in Figure 1Figure 3. In contrast, increasing the temperature reduces the tetrahedral structure in all four water models, which makes the distributions obtained with BLYP-D3 and SCAN qualitatively more similar to the distribution 
calculated with MB-pol at ambient temperature. It should be noted that this apparent better agreement with the MB-pol distribution is the result of fortuitous error cancellation associated with intrinsic deficiencies in the ability of BLYP-D3 and SCAN to represent water and not a consequence of NQEs, since when NQEs are explicitly taken into account in the simulations, all DFT models predict a significantly more tetrahedral structure. This behavior for all DFT models is exactly the opposite of the effect on the three-body correlations coming from a higher temperature.
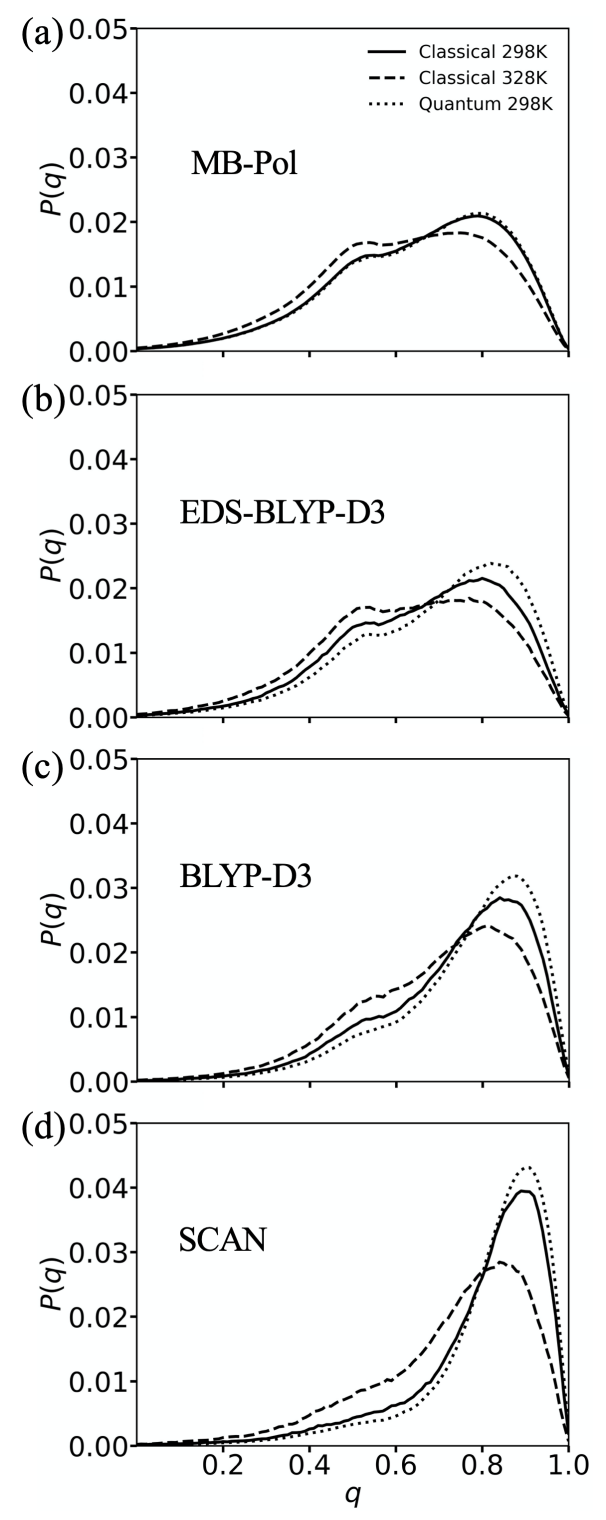

Figure 4. Tetrahedral order parameter $q$ distribution of (a) MB-Pol, (b) EDS-BLYP-D3, (c) BLYPD3 and (d) SCAN water model at $298 \mathrm{~K}$ with classical nuclei (solid) and with quantized nuclei (dotted), and at 328K (dashed). 
To determine how the additional thermal energy available at $328 \mathrm{~K}$ perturbs the system dynamics depending on the underlying water model, we computed the hydrogen-bond dynamics and water self-diffusion constant at both temperatures, with and without including NQEs. In this analysis, we consider that molecule $j$ is hydrogen-bonded to molecule $i$ if the $O_{i}-O_{j}$ distance is shorter than $3.5 \AA$ and the $\mathrm{H}_{i}-\mathrm{O}_{i}-\mathrm{O}_{j}$ angle is smaller than $30^{\circ}$, where $\mathrm{H}_{i}$ is one of the two bonded hydrogen atoms to $\mathrm{O}_{i}$. At a given time $t$, the hydrogen bond matrix is computed as

$$
h_{i j}=\left\{\begin{array}{ll}
1, & j \text { hydrogen }- \text { bonded to } i \\
0, & \text { otherwise }
\end{array},\right.
$$

The corresponding hydrogen-bond correlation function is defined as

$$
\langle h(0) h(\tau)\rangle=\frac{1}{N_{\text {wat }}\left(N_{\text {wat }}-1\right)} \sum_{i \neq j}\left\langle h_{i j}(0) h_{i j}(\tau)\right\rangle .
$$

In the analysis of the quantum simulations with the DFT models, we observe spurious water autoionization approximately $5 \%$ of the time. Due to the ambiguity of assigning bonded hydrogens to oxygens with auto-ionized water, the hydrogen bond matrix $h_{i j}$ is set to be the value at the closest time when the bonding topology is well defined, i.e., when water auto-ionization does not happen. This transient auto-ionization was also reported in Ref. ${ }^{45}$ and is likely the consequence of NQEs further reducing the proton transfer barrier between two water molecule which is already underestimated in the DFT models of water. ${ }^{46}$ As such, NQEs strengthen the hydrogen bonds and make DFT water more structured as shown in Figure 1Figure 4, which results in a slower hydrogen-bond dynamics as shown in Figure 5. As expected, simulations carried out at $328 \mathrm{~K}$ display an accelerated hydrogen-bond dynamics as seen in more rapid decays of the corresponding hydrogen-bond correlation functions, which is exactly the opposite of the effect of NQEs in these DFT models. In MB-pol, the NQEs have little effect on the h-bond dynamics, while the higher temperature speeds them up to a certain degree, as expected. 

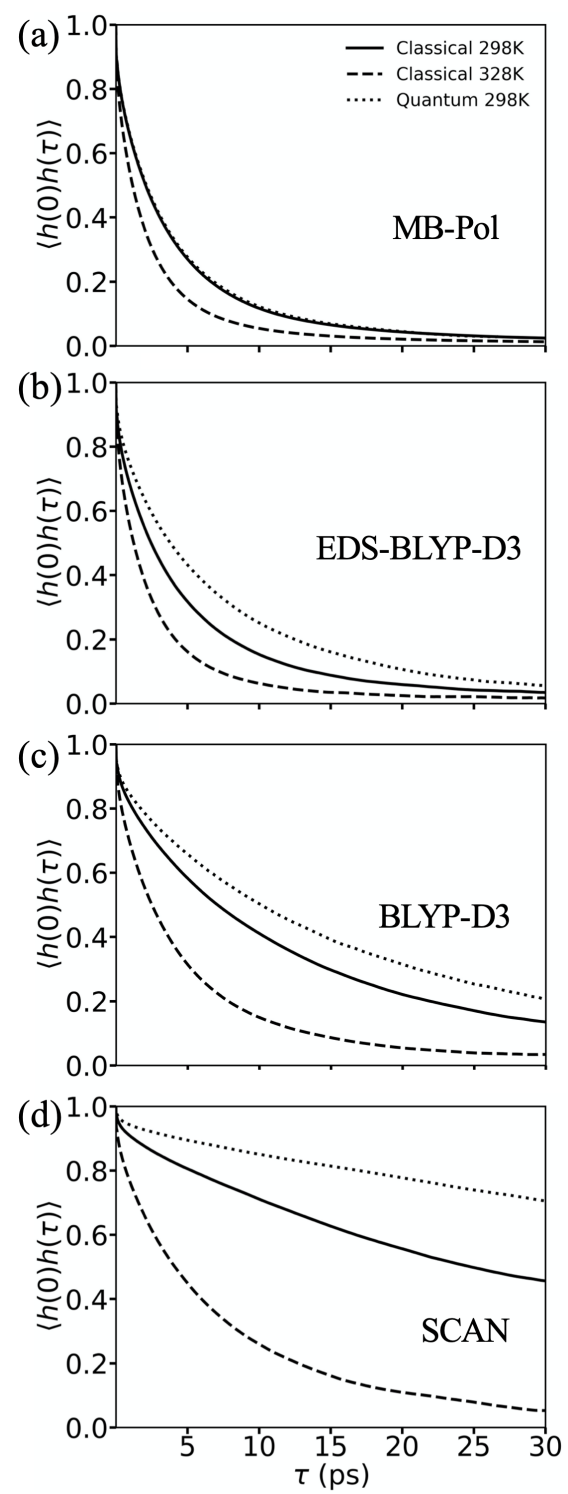

Figure 5. Hydrogen bond correlation function of (a) MB-Pol, (b) EDS-BLYP-D3, (c) BLYP-D3 and (d) SCAN water model at $298 \mathrm{~K}$ with classical nuclei (solid) and with quantized nuclei (dotted), and at $328 \mathrm{~K}$ (dashed).

We further investigated water dynamics by computing the self-diffusion constant from a linear fit to the 5 ps -15 ps segment of the mean-square displacement (MSD), defined as

$$
\operatorname{MSD}(t)=\left\langle\left(\boldsymbol{r}_{\mathrm{O}}(t)-\boldsymbol{r}_{\mathrm{O}}(0)\right)^{2}\right\rangle,
$$

where $\boldsymbol{r}_{\mathrm{O}}$ represents the oxygen position of a water molecule. This function is typically averaged over all water molecules in the homogeneous liquid system. The computed values are summarized 
in Table 1.We also report the diffusion constants after a correction for the finite simulation box used in simulations via ${ }^{47}$

$$
D(\infty)=D(L)+\frac{\xi k_{B} T}{6 \pi \eta L^{\prime}}
$$

where $\xi=2.837297$ is a constant for cubic boxes, $k_{B}$ is the Boltzmann constant, $T$ is the simulation temperature, and $L$ is the simulation box side length. In evaluating Eq. (5), the experimentally determined viscosity of water $\eta$ was used, ${ }^{48}$ which results in an overestimation of $D(\infty)$ for the BLYP-D3 and SCAN models since the over-structuring predicted by these two models would actually be associated with a viscosity higher than the experimental value (if the viscosity could be readily calculated from the AIMD, which it cannot). Clearly, for the three DFT models, the NQEs slows down the water self-diffusion while the elevated temperature, in an exactly opposite trend, accelerates the dynamics. For MB-pol, in spite of a slower hydrogen-bond dynamics, NQEs do not introduce a significant difference in the diffusion constant at ambient temperature, while, as expected, a much faster diffusion is observed at $328 \mathrm{~K}$.

Table 1. Self-diffusion constants of water in $\AA^{2} / p s$.

\begin{tabular}{|c|c|c|c|c|c|}
\hline Model & & MB-Pol & EDS-BLYP- & BLYP-D3 & SCAN \\
& & & D3 & & \\
\hline \multirow{2}{*}{$\begin{array}{c}\text { Diffusion } \\
\text { Constant }\end{array}$} & Classical 298K & $0.23 \pm 0.01$ & $0.19 \pm 0.03$ & $0.08 \pm 0.02$ & $0.03 \pm 0.02$ \\
\cline { 2 - 6 } & Classical 328K & $0.38 \pm 0.05$ & $0.32 \pm 0.03$ & $0.20 \pm 0.03$ & $0.14 \pm 0.04$ \\
\cline { 2 - 6 } & Quantum 298K & $0.23 \pm 0.05$ & $0.13 \pm 0.02$ & $0.06 \pm 0.03$ & $0.009 \pm 0.005$ \\
\hline $\begin{array}{c}\text { Diffusion } \\
\text { Constant after } \\
\text { Size Correction }\end{array}$ & Classical 298K & $0.27 \pm 0.01$ & $0.23 \pm 0.03$ & $0.12 \pm 0.02$ & $0.09 \pm 0.02$ \\
\cline { 2 - 6 } & Classical 328K & $0.42 \pm 0.05$ & $0.37 \pm 0.03$ & $0.25 \pm 0.03$ & $0.20 \pm 0.04$ \\
\cline { 2 - 6 } & Quantum 298K & $0.27 \pm 0.05$ & \multicolumn{5}{|c|}{$0.17 \pm 0.02$} & $0.10 \pm 0.03$ & $0.069 \pm 0.005$ \\
\hline Experimental & $298 \mathrm{~K}$ & $329 \mathrm{~K}$ & \multicolumn{5}{|c|}{0.44} \\
\cline { 2 - 6 } & \multicolumn{5}{|c|}{0.03} \\
\hline
\end{tabular}

Although it is not the main focus of this study to compare water models relative to the experimental data, it is worth noting that, among the four models considered in our analyses, MBpol provides the most accurate description of water at both temperatures, followed by the EDS corrected BLYP-D3 model. Both structural and dynamical properties of BLYP-D3 at $328 \mathrm{~K}$ are accidentally close to the experimental values determined at $298 \mathrm{~K}$ but not as the result of effectively mimicking NQEs. 


\section{CONCLUSIONS}

In this study, we systematically investigated the effect of an elevated temperature in simulating water with AIMD, with a particular focus on a suggested empirical relationship between higher temperature and nuclear quantum effects at room temperature. We considered three DFT-based AIMD water models, the widely used BLYP-D3 functional, with and without the EDS correction, and the meta-GGA SCAN functional. For all three DFT water models, the analysis of several structural and dynamical properties indicates that performing classical MD simulations at a higher temperature $(328 \mathrm{~K})$ introduces distinct and often opposite effects compared to performing quantum NQE simulations at $298 \mathrm{~K}$. For MB-pol, the elevated temperature seems to have a similar softening effect as NQEs at the two-body level, but the three-body correlation, hydrogen-bond dynamics, and diffusivity are clearly disrupted by the higher temperature. These findings suggest that "mimicking" NQEs in water by performing classical MD simulations at an elevated temperature is problematic and, in some cases, quite misleading. Importantly, we consistently

found stronger hydrogen bonds when NQEs are explicitly taken into account in simulations with all DFT-based water models considered in this study. This implies that the over-structuring exhibited by these particular DFT models is further emphasized by NQEs. We note, however, that performing classical MD simulations with DFT models at an elevated temperature accidentally reduces the over-structuring issue in an ad hoc way. Based on our analyses, we conclude that the elevated temperature approach does not represent a physically correct way of effectively mimicking NQEs and requires further careful characterization, including an examination of higher-order correlations and both molecular and collective dynamics, on a case-by-case basis, before being applied to other systems or to new water models (DFT-based or otherwise).

\section{ACKNOWLEDGEMENTS}

This research was supported in part by the U.S. Department of Energy (DOE), Office of Basic Energy Sciences, Division of Chemical Sciences, Geosciences, and Biosciences under Award Numbers DE-SC0018648 (G.A.V.) and DE-SC0019490 (F.P.). The research was also supported in part by the National Institute of General Medical Sciences (NIGMS) of the U.S. National Institutes of Health (NIH) through grant R01 GM053148 (G.A.V). Computational resources were 
provided by the Research Computing Center (RCC) at the University of Chicago. We thank Dr. Paul Calio for analysis tools, Dr. Xinyou Ma for SCAN setup, and Won Hee (Harry) Ryu for discussions.

\section{DATA AVAILABILITY}

The data that support the findings of this study are available from the corresponding author upon reasonable request.

\section{REFERENCES}

${ }^{1}$ M. Ceriotti, W. Fang, P. G. Kusalik, R. H. McKenzie, A. Michaelides, M. A. Morales, and T. E. Markland, Chem. Rev. 116, 7529 (2016).

${ }^{2}$ B. Chen, I. Ivanov, M. L. Klein, and M. Parrinello, Phys. Rev. Lett. 91, 215503 (2003).

${ }^{3}$ S. Habershon, T. E. Markland, and D. E. Manolopoulos, J. Chem. Phys. 131, 024501 (2009).

${ }^{4}$ J. A. Morrone, and R. Car, Phys. Rev. Lett. 101, 017801 (2008).

${ }^{5}$ O. Marsalek, and T. E. Markland, J. Phys. Chem. Lett. 8, 1545 (2017).

${ }^{6}$ R. P. Feynman, and A. R. Hibbs, Quantum mechanics and path integrals (McGraw-Hill, New York,, 1965), International series in pure and applied physics,

${ }^{7}$ T. E. Markland, and M. Ceriotti, Nat. Rev. Chem. 2, 1 (2018).

${ }^{8}$ T. E. Markland, and D. E. Manolopoulos, J. Chem. Phys. 129, 024105 (2008).

${ }^{9}$ S. J. Buxton, and S. Habershon, J. Chem. Phys. 147, 224107 (2017).

${ }^{10}$ M. Ceriotti, and D. E. Manolopoulos, Phys. Rev. Lett. 109, 100604 (2012).

${ }^{11}$ M. E. Tuckerman, J. Phys.: Condens. Matter 14, R1297 (2002).

${ }^{12}$ J. A. Pople, P. M. Gill, and B. G. Johnson, Chem. Phys. Lett. 199, 557 (1992).

${ }^{13}$ M. J. Gillan, D. Alfe, and A. Michaelides, J. Chem. Phys. 144, 130901 (2016).

${ }^{14}$ L. Hernández de la Peña, and P. G. Kusalik, J. Am. Chem. Soc. 127, 5246 (2005).

${ }^{15}$ F. Paesani, S. Iuchi, and G. A. Voth, J. Chem. Phys. 127, 074506 (2007).

${ }^{16}$ Y. Yao, and Y. Kanai, J. Chem. Phys. 153, 044114 (2020).

${ }^{17}$ Y. Yao, and Y. Kanai, J. Phys. Chem. Lett. 12, 6354 (2021).

${ }^{18}$ J. Sun, A. Ruzsinszky, and J. P. Perdew, Phys. Rev. Lett. 115, 036402 (2015).

${ }^{19}$ A. D. Becke, Phys. Rev. A 38, 3098 (1988).

${ }^{20}$ C. T. Lee, W. T. Yang, and R. G. Parr, Phys. Rev. B 37, 785 (1988).

${ }^{21}$ S. Grimme, J. Antony, S. Ehrlich, and H. Krieg, J. Chem. Phys. 132, 154104 (2010).

${ }^{22}$ P. B. Calio, G. M. Hocky, and G. A. Voth, J. Chem. Theory Comput. 16, 5675 (2020).

${ }^{23}$ V. Babin, C. Leforestier, and F. Paesani, J. Chem. Theory Comput. 9, 5395 (2013).

${ }^{24}$ V. Babin, G. R. Medders, and F. Paesani, J. Chem. Theory Comput. 10, 1599 (2014).

${ }^{25}$ G. R. Medders, V. Babin, and F. Paesani, J. Chem. Theory Comput. 10, 2906 (2014).

${ }^{26}$ S. K. Reddy, S. C. Straight, P. Bajaj, C. Huy Pham, M. Riera, D. R. Moberg, M. A. Morales, C. Knight, A. W. Götz, and F. Paesani, J. Chem. Phys. 145, 194504 (2016).

${ }^{27}$ F. Paesani, Acc. Chem. Res. 49, 1844 (2016).

${ }^{28}$ C. Hartwigsen, S. Godecker, and J. Hutter, Phys. Rev. B 58, 3641 (1998).

${ }^{29}$ J. VandeVondele, and J. Hutter, J. Chem. Phys. 118, 4365 (2003). 
${ }^{30}$ M. Chen, H.-Y. Ko, R. C. Remsing, M. F. C. Andrade, B. Santra, Z. Sun, A. Selloni, R. Car, M. L. Klein, and J. P. Perdew, Proc. Natl. Acad. Sci. U. S. A. 114, 10846 (2017).

${ }^{31}$ T. D. Kuhne, M. Iannuzzi, M. Del Ben, V. V. Rybkin, P. Seewald, F. Stein, T. Laino, R. Z. Khaliullin, O. Schutt, F. Schiffmann, D. Golze, J. Wilhelm, S. Chulkov, M. H. Bani-Hashemian, V. Weber, U. Borstnik, M. Taillefumier, A. S. Jakobovits, A. Lazzaro, H. Pabst, T. Muller, R. Schade, M. Guidon, S. Andermatt, N. Holmberg, G. K. Schenter, A. Hehn, A. Bussy, F. Belleflamme, G. Tabacchi, A. Gloss, M. Lass, I. Bethune, C. J. Mundy, C. Plessl, M. Watkins, J. VandeVondele, M. Krack, and J. Hutter, J. Chem. Phys. 152, 194103 (2020).

${ }^{32}$ J. VandeVondele, M. Krack, F. Mohamed, M. Parrinello, T. Chassaing, and J. Hutter, Comput. Phys. Commun. 167, 103 (2005).

${ }^{33}$ G. A. Tribello, M. Bonomi, D. Branduardi, C. Camilloni, and G. Bussi, Comput. Phys. Commun. 185, 604 (2014).

${ }^{34}$ S. Lehtola, C. Steigemann, M. J. T. Oliveira, and M. A. L. Marques, Softwarex 7, 1 (2018).

${ }^{35}$ H. Wang, L. Zhang, J. Han, and E. Weinan, Comput. Phys. Commun. 228, 178 (2018).

${ }^{36}$ L. Zhang, J. Han, H. Wang, W. A. Saidi, and R. Car, arXiv preprint arXiv:1805.09003 (2018).

${ }^{37}$ S. Plimpton, J. Comput. Phys. 117, 1 (1995).

${ }^{38}$ V. Kapil, M. Rossi, O. Marsalek, R. Petraglia, Y. Litman, T. Spura, B. Cheng, A. Cuzzocrea, R. H. Meißner, and D. M. Wilkins, Comput. Phys. Commun. 236, 214 (2019).

${ }^{39}$ M. Rossi, M. Ceriotti, and D. E. Manolopoulos, J. Chem. Phys. 140, 234116 (2014).

${ }^{40}$ S. K. Reddy, D. R. Moberg, S. C. Straight, and F. Paesani, J. Chem. Phys. 147, 244504 (2017).

${ }^{41}$ L. B. Skinner, C. Huang, D. Schlesinger, L. G. Pettersson, A. Nilsson, and C. J. Benmore, J. Chem. Phys. 138, 074506 (2013).

${ }^{42}$ A. Soper, Chem. Phys. 258, 121 (2000).

${ }^{43}$ A. Soper, and C. Benmore, Phys. Rev. Lett. 101, 065502 (2008).

${ }^{44}$ P.-L. Chau, and A. Hardwick, Mol. Phys. 93, 511 (1998).

${ }^{45}$ F. Giberti, A. A. Hassanali, M. Ceriotti, and M. Parrinello, J. Phys. Chem. B 118, 13226 (2014).

${ }^{46}$ G. Cassone, J. Phys. Chem. Lett. 11, 8983 (2020).

${ }^{47}$ I.-C. Yeh, and G. Hummer, J. Phys. Chem. B 108, 15873 (2004).

${ }^{48}$ M. Holmes, N. Parker, and M. Povey, in Journal of Physics: Conference Series (IOP Publishing, 2011), p. 012011.

${ }^{49}$ M. Holz, S. R. Heil, and A. Sacco, Phys. Chem. Chem. Phys. 2, 4740 (2000). 